\title{
Seizures and Epilepsy in Times of Corona Virus Disease 2019 Pandemic
}

\section{Review}

Journal of Epilepsy Research pISSN 2233-6249 / elSSN 2233-6257

Received May 9, 2020

Revised May 22, 2020

Accepted June 5, 2020

Corresponding author:

Manjari Tripathi, MD, DM

Department of Neurology, All India

Institute of Medical Sciences, Room No 705,

Neurosciences Centre, New Delhi 110029,

India

Tel. +9868398269

Fax. +91-26588248

E-mail; mantriaiims@gmail.com

\author{
Jasmine Parihar, MD, DM', Manjari Tripathi, MD, DM², Rajinder K. Dhamija, MD, DNB, FRACP \\ ${ }^{1}$ Department of Neurology, Lady Hardinge Medical College \& Associated Hospitals, New Delhi; ${ }^{2}$ Department of \\ Neurology, All India Institute of Medical Sciences, New Delhi, India
}

\begin{abstract}
The end of the year 2019 was marked by novel coronavirus (severe acute respiratory syndrome coronavirus-2, SARS-CoV-2) outbreak in China that rapidly spread to the rest of the world. While the involvement of the lower respiratory system causing pneumonia is identified as the primary target of the virus, extra-pulmonary manifestations, especially of the central nervous system, are also being increasingly reported. Previous research on Middle East respiratory syndrome coronavirus and SARS-CoV have shown neurological involvement in human coronavirus infections. While several cases of seizures have been reported in patients with coronavirus disease 2019 (COVID-19) patients, there is no specific data to suggest an association of COVID-19 with epilepsy. Epilepsy patients on immunosuppressive medications may have a higher risk of contracting the viral infection. There can be an indirect relation of COVID-19 to epilepsy as the viral infection is associated with fever in most COVID-19 cases, which can lower seizure threshold. Additionally, inadequate sleep and stress due to ongoing pandemic of coronavirus can be another trigger for seizure precipitation in epilepsy patients. Drug compliance, availability of antiepileptic drugs, and drug interactions with COVID-19 experimental drugs are major concerns in epilepsy patients. Adopting telemedicine services and the use of epilepsy helplines may be important in assisting epilepsy patients and ensuring that treatment continues uninterrupted. (2020;10:3-7)
\end{abstract}

Key words: Coronavirus, SARS virus, Epilepsy, Pandemics

\section{Introduction}

Coronaviruses belongs to a family of RNA viruses that typically cause mild respiratory disease in humans. However, two highly pathogenic human coronaviruses - severe acute respiratory syndrome coronavirus (SARS-CoV) and Middle East respiratory syndrome coronavirus (MERS-CoV) - emerged from animal reservoirs to cause outbreaks and epidemic in the past. MERS-CoV was responsible for the MERS that occurred in 2012, whereas SARS-CoV caused an outbreak of SARS in 2003. The end of the year 2019 was marked by another novel coronavirus outbreak, severe acute respiratory syndrome coronavirus-2 (SARS-CoV-2), first documented in Wuhan, Hubei Province, China. ${ }^{1}$ As of May 6, 2020, more than 3.7 million cases of Corona virus Disease 2019 (COVID-19) have been reported worldwide, resulting in more than 258,361 deaths. In India, more than 50,000 cases and more than 1,500 deaths have been hitherto reported (https://www.mygov.in/covid-19; last accessed May 6, 2020).

While the lower respiratory system causing pneumonia is identi- fied as the primary target of the virus, extra-pulmonary manifestations, especially of the central nervous system (CNS), are also being increasingly reported. SARS-CoV-2 shows opportunistic neurotropism by escaping the immune response and interacting with the angiotensin-converting enzyme 2 receptor to gain entry inside the neural tissue. ${ }^{2}$ It is proposed that these viruses may gain access to the CNS either through the hematogenous or retrograde neuronal route like olfactory, trigeminal and vagal nerves., ${ }^{3,4}$ The most severe neurological manifestations associated with COVID-19 include impaired consciousness, acute ischemic stroke, cerebral venous sinus thrombosis, cerebral hemorrhage, acute hemorrhagic necrotizing encephalopathy, and Guillain-Barre syndrome. ${ }^{5-7}$ Other neurological manifestations include headache, dizziness, confusion, hypogeusia, hyposmia, and muscle injury. ${ }^{5,68-13}$ The neurological manifestations of COVID-19 could be related to the direct CNS infection by SARS-CoV-2 or due to systemic complications. This article reviews the association of epilepsy and seizures with COVID-19.

In a large retrospective study of 1,099 patients with labo- 
ratory-confirmed COVID-19 in China by Guan et al., ${ }^{14}$ no neurological involvement was reported. However, in the series by Mao et al., ${ }^{12} 34.6 \%$ of 214 patients had associated neurological signs or symptoms, with only one patient having a seizure in this series. This patient was in the "severe group", categorized according to the severity of the respiratory symptoms.

There are case reports of the occurrence of seizures in COVID-19 patients. Patients may present with seizures due to viral encephalitis or encephalopathies related to metabolic disturbances, hypoxia, and renal or hepatic dysfunction due to complications of COVID-19 disease. Multiple generalized tonic-clonic seizures with normal brain magnetic resonance imaging (MRI) have been reported in a 30-year-old COVID-19 infected female who started with respiratory symptoms three days before the seizures. ${ }^{15}$ Another 78 -year-old SARS-CoV-2 infected female patient had presented with focal myoclonic status epilepticus (SE). ${ }^{16}$ She had preexisting well-controlled epilepsy as a sequel to herpes simplex virus-1 encephalitis that developed 2 years prior to the current presentation with MRI brain showing focal gliosis. She later developed respiratory symptoms and was tested positive for COVID-19, though she recovered well after conservative management.

Previous research on MERS-COV and SARS-CoV have also shown neurological involvement in human coronavirus infections. In a study of 70 patients with MERS-CoV infection, 26\% had altered mental status and $9 \%$ had seizures. ${ }^{17}$ SARS-CoV RNA has been detected in the serum and cerebrospinal fluid of two patients with SARS complicated by SE. ${ }^{18,19}$

While seizures have been reported in COVID-19 patients, there is no data to suggest an association of COVID-19 with epilepsy. There is one case report of COVID-19 in a known epilepsy patient, in which the patient did not experience any clinical seizure in the course of infection. ${ }^{20}$ The cause of epilepsy is not specified in this report. Despite the current lack of evidence of any association between COVID-19 and epilepsy, there are considerations of potential links between the two. Epilepsy per se is not a high-risk condition for the development of COVID-19 infection. However, epilepsy patients on immunosuppressive medications like steroids, adrenocorticotropic hormone, intravenous immunoglobulins, or everolimus used in tuberous sclerosis may have a higher risk of contracting the infection.

The coincidence of epilepsy and COVID-19 in a patient can have some impact on seizure control in patients as the infection is associated with fever in 60-98\% of COVID-19 cases. ${ }^{10,12,14}$ Increase in temperature risks precipitating seizure in many epilepsy patients, particularly those with Dravet syndrome who can have an aggravation of seizures with increased body temperature or stress. COVID-19 infection has also been associated with psychological and sleep disturbances. Inadequate sleep is another trigger of precipitation of seizure in generalized epilepsies like Juvenile myoclonic epilepsy. Patients with epilepsy not infected with COVID also experience psychological stress and altered sleep cycle owing to lockdown and hence, alteration in daily routine, which can result in precipitation of seizure. In a web-based cross-sectional survey by Huang and Zhao, ${ }^{21} 18.2 \%$ of 7,236 self-selected volunteers experienced poor sleep quality during the COVID-19 outbreak in China. Severe psychological distress has recently been reported to be more common among 252 epilepsy patients during the COVID-19 outbreak in southwest China when compared to normal subjects. ${ }^{22}$

\section{Sudden unexpected death in epilepsy (SUDEP) and COVID}

There is no data on the occurrence of SUDEP in epilepsy co-occurring with COVID-19 infection. An indirect relationship may exist as there is an increase in stress in COVID infected and un-infected epilepsy patients, and stress is a known risk factor for SUDEP. ${ }^{23}$ Viral infections have been implicated in relationship to SUDEP in the past. Wang et al. ${ }^{24}$ described a case of SUDEP associated with human herpesvirus-6 in an adolescent with tuberous sclerosis.

\section{Other challenges}

\section{Drug compliance}

Most countries are currently under lockdown to reduce the spread of the coronavirus and India also imposed lockdown on March 22, 2020. The current situation does not show any forthcoming relaxation. This has led to the restriction of routine or non-urgent outpatient facilities for patients in hospitals. Patients may also be experiencing difficulties in reaching pharmacies for refill prescriptions. Due to suddenly imposed lockdown, it is likely that a number of patients may have run out of their medications and experience breakthrough seizures. During the 2003 SARS outbreak, many patients avoided visiting hospitals because they feared infection from the virus. Lai et al. ${ }^{25}$ studied drug compliance and seizure frequency in these patients and found that 49 out of 227 cases stopped taking medication during the outbreak. Among them, 28 patients suffered seizure attacks when antiepileptic drugs (AED) were discountinued. Four cases developed cluster attacks re- 
quiring emergency room treatment and two cases had SE requiring admission in the intensive care unit (ICU). The notable risk factors for breakthrough seizures in this study were symptomatic etiologies, polytherapy, and non-seizure free before AED withdrawal.

\section{Shortage of AEDs}

The availability of AEDs is another challenge. As the pharmaceutical industry is heavily dependent on the import and export of raw materials to manufacture drugs including AEDs, the supply of several drugs to the market is affected. This is particularly important for the supply of rare drugs like vigabatrin, which is procured by local suppliers on a demand basis.

\section{Drug interactions}

The COVID-19 outbreak has put pressure on the health care systems to expedite clinical trials of experimental drugs against this virus. These drugs include lopinavir/ritonavir, remdesivir, favipiravir, ribavarin, chloroquine, and hydroxychloroquine. ${ }^{26}$ These drugs might become available for use shortly, which depends on the trial results. Hydroxychloroquine has been used in various parts of the world including India as prophylaxis for coronavirus infection. The epileptologists and physicians must be aware of drug interactions of AEDs with these experimental drugs. Carbamazepine, phenytoin, phenobarbitone, and primidone should not be co-administered with most of these experimental drugs due to concerns of decrease in levels of the COVID drugs. ${ }^{27,28}$ Chloroquine and hydroxychloroquine are contraindicated in patients taking lacosamide and cenobamate, given the common side effect of prolongation of QT interval and risk of cardiac arrhythmias, including Torsades de Pointes with these drugs. ${ }^{28}$ Interactions between antibiotics prescribed for the management of respiratory tract infections (azithromycin, quinolones and macrolides) and the COVID-19 experimental drugs should also be considered.

\section{Delay in workup}

Since the government and health care centers recommended limiting non-essential care during the pandemic, non-emergency epilepsy care may likely suffer from this situation because of delays in investigations like imaging and electroencephalography. Additionally, drug-refractory epilepsy patients planned for pre-surgical evaluations or epilepsy surgeries will have an even longer waiting period. ${ }^{29}$

\section{Management of epilepsy during COVD-19}

\section{Telemedicine}

Telemedicine is the practice of caring for patients remotely when the provider and patient are not physically present with each other. This may be through any agency such as via text message, phone call, or video call. Telemedicine, particularly video consultations, have been promoted and scaled up during the pandemic. In India, the guidelines for telemedicine have been issued by the ministry of health and family welfare in collaboration with NITI Aayog and Board of Governors Medical Council of India (available at https://www.mohfw.gov.in/pdf/Telemedicine.pdf) and the physician should follow the local guidelines while implementing telemedicine. However, the use of telemedicine cannot be overemphasized as a majority of the Indian patient population is not computer savvy and lacks an understanding of application of telemedicine services. For all these patients, a simple phone call on their mobile phones may be an excellent way of consultation and follow up. ${ }^{30}$

\section{Prescriptions}

To ensure the patients adherence to and availability of medications, the physician should prescribe the maintenance medication for a longer duration, preferably at least three months. The decision to taper the AEDs in patients who are well controlled or in remission should be postponed. The rescue medications should be prescribed and patients and their caregivers should be guided about the measures to deal with seizure occurrence.

\section{Fever and epilepsy concerns}

Epilepsy patients with COVID-19 (or any form of febrile illness) should also be instructed to have a low threshold for using an antipyretic (preferably paracetamol rather than aspirin) to prevent provoking seizures. In patients with fever sensitive seizures like Dravet's syndrome, increasing the dose of AEDs or adding an extra AED like clobazam can be considered during the febrile illness. American epilepsy society also recommends limiting the use of products that contain decongestants and herbal products like Ma Huang (ephedra), which have been used in treating COVID-19, as these products could exacerbate seizures. ${ }^{28}$

\section{Helplines}

Patients should be encouraged to use epilepsy helpline and support groups for the management of stress and guidance on their 
treatment.

\section{Emergency management of seizures}

In times of COVID-19, there can be an increase of patients presenting with seizures in the emergency setting. In addition to the neurological causes, proper history must be taken to rule out psychogenic non-epileptic seizures and alcohol and drug withdrawal seizures. These are expected to escalate due to an increase in stress and difficulty in accessing alcohol and other recreational drugs during lockdown. Patients presenting with SE should be managed as per the standard guidelines. However, it has been suggested to consider a SE management protocol which avoids sedation, if possible, to decrease the duration of ICU stay in the situation with constrained resources in COVID-19 times. $^{31}$

\section{Conclusion}

To conclude, there is no direct correlation of COVID-19 with epilepsy, though the relevant data is still emerging in COVID-19 infected patients. In lower-middle-income countries like India, particularly in the rural population, there is already a large treatment gap in epilepsy. The COVID-19 pandemic poses an additional burden which would worsen the already grim situation for these patients. All possible measures should be taken to provide easy health care access to patients through various ways such as telecommunication. Moreover, patient and caregiver education from epilepsy support groups can decrease the burden on health care facilities.

\section{References}

1. Zhu N, Zhang $D$, Wang $W$, et al. A novel coronavirus from patients with pneumonia in China, 2019. N Engl J Med 2020;382:727-33.

2. Baig AM, Khaleeq A, Ali U, Syeda H. Evidence of the COVID-19 virus targeting the CNS: tissue distribution, host-virus interaction, and proposed neurotropic mechanisms. ACS Chem Neurosci 2020;11:995-8.

3. Wu $Y, X u X$, Chen $Z$, et al. Nervous system involvement after infection with COVID-19 and other coronaviruses. Brain Behav Immun 2020;87:18-22.

4. Desforges $M$, Le Coupanec $A$, Dubeau $P$, et al. Human coronaviruses and other respiratory viruses: underestimated opportunistic pathogens of the central nervous system? Viruses 2019;12:14.

5. Li $Y$, Li $M$, Wang $M$, et al. Acute cerebrovascular disease following COVID-19: a single center, retrospective, observational study. Stroke Vasc Neurol 2020 Jul 2. doi: 10.1136/svn-2020-000431.

6. Zhao H, Shen D, Zhou H, Liu J, Chen S. Guillain-Barré syndrome associated with SARS-CoV-2 infection: causality or coincidence? Lancet
Neurol 2020;19:383-4.

7. Poyiadji N, Shahin G, Noujaim D, Stone M, Patel S, Griffith B. COVID-19-associated acute hemorrhagic necrotizing encephalopathy: CT and MRI features. Radiology 2020;296:E119-20.

8. Asadi-Pooya AA, Simani L. Central nervous system manifestations of COVID-19: a systematic review. J Neurol Sci 2020;413:116832.

9. Wang D, Hu B, Hu C, et al. Clinical characteristics of 138 hospitalized patients with 2019 novel coronavirus-infected pneumonia in Wuhan, China. JAMA 2020;323:1061-9.

10. Yang $X, Y u Y, X u$ J, et al. Clinical course and outcomes of critically ill patients with SARS-CoV-2 pneumonia in Wuhan, China: a single-centered, retrospective, observational study. Lancet Respir Med 2020;8:475-81.

11. Chen $N$, Zhou M, Dong $X$, et al. Epidemiological and clinical characteristics of 99 cases of 2019 novel coronavirus pneumonia in Wuhan, China: a descriptive study. Lancet 2020;395:507-13.

12. Mao $L$, Jin $H$, Wang $M$, et al. Neurologic manifestations of hospitalized patients with coronavirus disease 2019 in Wuhan, China. JAMA Neurol 2020;77:1-9.

13. Xydakis MS, Dehgani-Mobaraki P, Holbrook EH, et al. Smell and taste dysfunction in patients with COVID-19. Lancet Infect Dis 2020;20: 1015-6.

14. Guan WJ, Ni ZY, Hu Y, et al. Clinical characteristics of coronavirus disease 2019 in China. N Engl J Med 2020;382:1708-20.

15. Karimi N, Sharifi Razavi A, Rouhani N. Frequent convulsive seizures in an adult patient with COVID-19: a case report. Iran Red Crescent Med J 2020;22:e102828.

16. Vollono C, Rollo $E$, Romozzi $M$, et al. Focal status epilepticus as unique clinical feature of COVID-19: a case report. Seizure 2020;78:109-12.

17. Saad M, Omrani AS, Baig K, et al. Clinical aspects and outcomes of 70 patients with Middle East respiratory syndrome coronavirus infection: a single-center experience in Saudi Arabia. Int I Infect Dis 2014;29: 301-6.

18. Hung EC, Chim SS, Chan PK, et al. Detection of SARS coronavirus RNA in the cerebrospinal fluid of a patient with severe acute respiratory syndrome. Clin Chem 2003;49:2108-9.

19. Lau KK, Yu WC, Chu CM, Lau ST, Sheng B, Yuen KY. Possible central nervous system infection by SARS coronavirus. Emerg Infect Dis 2004; 10:342-4.

20. Yasri S, Wiwanikit V. COVID-19 and epilepsy. Ann Indian Acad Neurol 2020;23 Suppl S1:43.

21. Huang Y, Zhao N. Generalized anxiety disorder, depressive symptoms and sleep quality during COVID-19 outbreak in China: a web-based cross-sectional survey. Psychiatry Res 2020;288:112954.

22. Hao $X$, Zhou $D$, Li Z, et al. Severe psychological distress among patients with epilepsy during the COVID-19 outbreak in southwest China. Epilepsia 2020;61:1166-73.

23. Sveinsson $O$, Andersson $T$, Mattsson $P$, Carlsson $S$, Tomson T. Clinical risk factors in SUDEP: a nationwide population-based case-control study. Neurology 2020;94:e419-29.

24. Wang J, Huff K, McMasters R, Cornford ME. Sudden unexpected death 
associated with HHV-6 in an adolescent with tuberous sclerosis. Pediatr Neurol 1999;21:488-91.

25. Lai SL, Hsu MT, Chen SS. The impact of SARS on epilepsy: the experience of drug withdrawal in epileptic patients. Seizure 2005;14:557-61.

26. Singh AK, Singh A, Shaikh A, Singh R, Misra A. Chloroquine and hydroxychloroquine in the treatment of COVID-19 with or without diabetes: a systematic search and a narrative review with a special reference to India and other developing countries. Diabetes Metab Syndr 2020;14: 241-6.

27. University of Liverpool. COVID-19 Interaction. Interactions with Experimental COVID-19 Therapies Interactions with Experimental COVID-19 Therapies [Internet]. Liverpool: University of Liverpool, [cited 2020 May 6]. Available at: https://uww.covid19-druginteractions.org.
28. Welty T, Cokley J, Gidal B. Managing Patients with Epilepsy during COVID-19 Pharmacotherapy-related Recommendations [Internet]. Chicago: American Epilepsy Society, [cited 2020 May 6]. Available at: https://uww.aesnet.org/about_aes/position_statements/covid-19/deliver y-of-care/covid-pharmacotherapy-epilepsy.

29. Schaller K. Neurosurgeons in the corona crisis: striving for remedy and redemption. A message from the president of the EANS. Acta Neurochir (Wien) 2020;162:1217-9.

30. Bahrani $K$, Singh MB, Bhatia $R$, et al. Telephonic review for outpatients with epilepsy-A prospective randomized, parallel group study. Seizure 2017;53:55-61.

31. Kinney MO, Brigo F, Kaplan PW. Optimizing status Epilepticus care during the COVID-19 pandemic. Epilepsy Behav 2020;109:107124. 